\title{
¿Debe el Estado financiar las artes y la cultura? Revisión de literatura ${ }^{1}$
}

\author{
Luis Antonio Palma Martos ${ }^{2}$ \\ Luis Fernando Aguado Quintero ${ }^{3}$
}

\section{Resumen}

En el artículo se revisa una amplia bibliografía acerca del conjunto de argumentos para justificar el apoyo con fondos públicos a las artes y la cultura. Desde la presencia de condiciones particulares en la oferta ("enfermedad de los costes"), o en la demanda (bienes públicos), la existencia de preferencias comunitarias (bien de mérito), hasta la aportación a la actividad económica y al empleo (Estudios de Impacto Económico de la Cultura). Se señalan las limitaciones que presenta cada argumento y aquellos que pueden resultar convincentes desde la teoría económica.

Palabras clave: Economía de la cultura; Política cultural; Enfermedad de los costes de Baumol; Derechos de autor.

\begin{abstract}
Should the government support arts and culture? A literature review

This article will review an extensive bibliography concerning the complete set of arguments justifying the use of public funds to support Arts and Culture. Particular conditions regarding the supply ("the cost disease") and the demand (public goods), the existence of community preferences (merit good), and the contribution to economic activity and employment (Studies of the Economic Impact of Culture) are all taken into consideration. The limitations of each argument are pointed out, as well as those that gain strength based on economic theory.
\end{abstract}

Keywords: Cultural economics; Cultural policy; Baumol's cost-disease; Copyright. JEL Z11, O34.

\section{Introducción}

La "Economía de la Cultura" constituye un área de especialización reciente de la economía (Throsby, 1994; Ginsburgh; Throsby, 2006). ${ }^{4}$ Frey (2000)

(1) Trabalho recebido em agosto de 2009 e aprovado em novembro de 2009.

(2) Profesor Titular de Universidad de Sevilla, Departamento de Economía e Historia Económica, Sevilla, España. E-mail: Ipalma@us.es.

(3) Asistente Honorario, Departamento de Economía e Historia Económica. Universidad de Sevilla, España / Profesor Asistente (en comisión de estudios), Departamento de Economía, Pontificia Universidad Javeriana Cali, Colombia. E-mail: 1faguado@us.es; 1faguado@javerianacali.edu.co.

(4) En 1991 el sistema de clasificación del Journal of Economic Literature (JEL) sitúa la producción intelectual en el epígrafe Z -otros tópicos especiales-, bajo el sub-epígrafe Z1: Cultural Economics. En 2001, se introduce el sub-epígrafe Z11: Economics of the Arts and Literature (Towse, 2005, p.F263). En 2006 se publica el primer Handbook of the Economics of Art and Culture (Ginsburgh; Throsby, 2006) en la reconocida serie de manuales editada por Kenneth Arrow y Michael Intriligator. En 2008 aparece la primera entrada en The New Palgrave Dictionary of Economics; "art, economics of", (Throsby, 2008). La Association for Cultural Economics International (www.culturaleconomics.org) es creada en 1973 y desde 1979 hasta la actualidad ha celebrado 16 reuniones bianuales. Por último, el Journal of Cultural Economics aparece en forma ininterrumpida desde 1977. 
y Towse (2003) la han definido como la aplicación de la teoría y herramientas de la economía al análisis de la organización económica del sector cultural (artes escénicas, artes visuales, patrimonio, industrias culturales) y del comportamiento de quiénes producen y consumen los bienes y servicios culturales, incluyendo el papel del gobierno. La política cultural ocupa un lugar central dentro de la "Economía de la Cultura". Sus ámbitos se reflejan en leyes, normas y programas, para promover la generación, acceso y difusión de valores y experiencias culturales por parte de los gobiernos, las empresas y los individuos (Mulcahy, 2006). Cubre temas tan variados como la financiación de museos, orquestas sinfónicas y bibliotecas, la estrategia de preservación del patrimonio histórico, la promoción de la música folclórica y festivales populares, entre muchas actividades. Para ello cuenta con diversos instrumentos tales como subsidios, deducciones fiscales y gasto público (producción directa); leyes y normas (calidad, protección del patrimonio, copyright); ayudas (becas, cursos, premios); el mecenazgo y la filantropía.

La política cultural es una realidad y con esto la participación del Estado en la financiación. De hecho, muchos museos son de propiedad del Estado y gestionados por éste, en consecuencia el coste de sus entradas es subsidiado, lo mismo sucede con el mantenimiento y la operación del patrimonio cultural (sitios históricos, bibliotecas, palacios, casco histórico de las ciudades); los teatros, las orquestas sinfónicas, la ópera, las compañías de danza, reciben a menudo ayuda del Estado en forma de subsidios y desgravaciones fiscales; también podemos señalar la existencia de una protección especial para el producto de estas actividades, como el derecho de autor (copyright).

Al observar esta realidad, los interrogantes que surgen son varios: ¿por qué una política cultural? ¿es necesaria la intervención del Estado en los mercados artísticos y culturales?, de ser necesaria la intervención del Estado, ¿cuál sería la mejor forma de hacerlo? En este contexto, en el artículo se analiza un aspecto clave de la política cultural: la financiación del arte y la cultura, en especial el debate sobre la financiación pública.

En la revisión de la literatura, se encuentra un relativo consenso en las siguientes acciones específicas de política cultural enfocadas en la financiación pública: el fomento a la educación artística en escuelas públicas con el objetivo de generar capital de consumo cultural a temprana edad; los subsidios a la demanda (para la compra de obras de artistas jóvenes en artes visuales y cubrir las "sillas vacías" de nuevas obras en artes escénicas); el valor de opción para justificar el legado intergeneracional, en especial para el patrimonio (mantenimiento de sitios históricos, grandes monumentos, bibliotecas y museos). En los sectores asociados a las nuevas tecnologías de la información y las comunicaciones, los derechos de 
autor (copyright) significan una alternativa de financiación, pese a la paradoja que implica, por lo que el tema sigue abierto a discusión.

Tras esta introducción, el artículo se organiza de la siguiente manera, en la primera sección, se analizan los argumentos a favor y sus críticas sobre la financiación pública del arte y la cultura. En la segunda sección, se presentan los argumentos sobre los cuales hay relativo consenso para justificar la participación directa del Estado en el arte y la cultura. La tercera sección, amplía el espectro de los instrumentos de la política cultural, concentrados básicamente en las ayudas directas (subsidios) e indirectas (exenciones fiscales), hacia el tema de la regulación, en especial la introducción de los derechos de autor (copyright), como elemento de la política cultural. En las últimas secciones, se presentan las conclusiones y la bibliografía consultada, respectivamente.

\section{Revisión de literatura}

El tema de la financiación de las artes y la cultura ha sido uno de los más sensibles en la subdisciplina de la "Economía de la Cultura". Tres citas sugestivas al respecto nos ilustran la afirmación:

El aspecto de la política que con mayor frecuencia ha suscitado la atención de los economistas es por qué las artes no pueden financiarse a sí mismas y por qué no debería esperarse que así fuera (Grampp, 1989, p. 20).

Gracias a la economía del arte se hace posible para el economista investigar la cuestión que inspira tanta curiosidad y levanta tanta polémica, de cuál sería la mejor manera de que las autoridades públicas promocionaran el arte (Frey, 2000, p. 121)

¿Por qué en una sociedad en la que sus gobernantes deben facilitar el bienestar de sus ciudadanos, ciertos productos, como los cuadros, o servicios, como las representaciones de ópera, requieren un apoyo financiero cuando la mayoría de los bienes y servicios generados en la economía dependen de su propia suerte en el mercado? (Baumol, 2003, p. 272).

Importantes economistas han contribuido al debate sobre esta cuestión. Desde Adam Smith, para quien las artes debían dejar su financiación a la iniciativa privada, hasta Robbins, Marshall, Keynes y Baumol para quienes el Estado debe jugar un papel preponderante en su auspicio. Siguiendo a Klamer, Petrova y Mignosa (2006) el arte y la cultura disponen de tres fuentes principales de financiación (ver Cuadro 1): a) el gobierno, b) el mercado, c) las organizaciones sin ánimo de lucro. Las distintas fuentes difieren de acuerdo con el país que se trate y, a su vez, generan su propio diseño institucional con respecto a la forma y los instrumentos empleados para llevar a cabo la financiación. 
Cuadro 1

Fuentes de financiación de las actividades culturales

\begin{tabular}{|l|l|l|l|}
\hline Fuente & \multicolumn{1}{|c|}{$\begin{array}{c}\text { Instrumentos } \\
\text { Girectos: Subsidios; } \\
\text { ayudas (becas, } \\
\text { concursos); provisión } \\
\text { directa (ejemplo: } \\
\text { propiedad de museos, } \\
\text { orquestas sinfónicas, } \\
\text { teatros) }\end{array}$} & $\begin{array}{l}\text { Criterios } \\
\text { Indirectos: Beneficios } \\
\text { calidad a seguir por los } \\
\text { artistas impuestos por } \\
\text { los Consejos de Artes } \\
\text { donde los servidores } \\
\text { públicos toman las } \\
\text { decisiones. Bienes } \\
\text { fúblicos, bienes de }\end{array}$ & $\begin{array}{l}\text { Concentración en la alta } \\
\text { cultura y en } \\
\text { organizaciones } \\
\text { culturales consolidadas. }\end{array}$ \\
\hline \multirow{2}{*}{ Mérito. }
\end{tabular}

Fuente: Elaboración propia con base en Klamer, Petrova y Mignosa (2006).

En efecto, la participación del Estado en la financiación de las artes tiene diversos matices. En los EE.UU. fue marginal hasta la creación del National Endowment for the Arts y, tradicionalmente, el sector privado (mercado) y las donaciones (mecenazgo) de particulares han jugado un importante papel. ${ }^{5}$ Lo contrario sucede en Europa Continental, donde el Estado participa fuertemente en el apoyo a las artes a través del gasto público (subsidios y la provisión directa).

Así, es como, en EE.UU. las deducciones por donaciones individuales, las exenciones en el impuesto sobre la propiedad y las desgravaciones en las ganancias de capital, son los principales instrumentos fiscales de ayuda al sector cultural, en particular, para las artes escénicas y los museos. ${ }^{6}$ En el caso de Europa, son el gasto público directo y el trato preferencial en el IVA para los

(5) En este sentido, en EE.UU. ha habido amplio debate sobre si destinar mayores fondos públicos a las artes puede significar un efecto de desplazamiento (crowding-out effect) de las donaciones privadas. Al respecto, Borgonovi (2006) encuentra, para el caso de los teatros en EE.UU., que en determinados casos el apoyo público y privado se complementan y se sustituyen dependiendo del tamaño del apoyo del gobierno, si los fondos representan un aumento o disminución con respecto a sus niveles anteriores y el nivel del agente que autoriza el gasto (gobierno federal, estatal o local).

(6) Más, en general, se debe decir que estos beneficios cubren a Instituciones Beneficias sin ánimo de Lucro, figura institucional dentro de la cual están circunscritas muchas de las manifestaciones culturales (ver Netzer, 2003). 
productos del sector (O'Hagan, 2003). Existen diferencias en los efectos de emplear el gasto público directo y los beneficios fiscales sobre el apoyo al sector cultural (Frey, 1999; 2003), en particular, sobre la magnitud de la ayuda y la manifestación cultural que la recibe. En el primer caso, la decisión sobre la cuantía y los beneficiarios corresponde al sector público, es decir, a la burocracia estatal. En el segundo caso, dicha decisión corresponde a los individuos y las empresas.

En la siguiente subsección, hacemos referencia a la política cultural, en concreto a la financiación pública, desde la perspectiva del pensamiento económico, y después planteamos los argumentos desde el análisis económico.

\subsection{El pensamiento económico y la política cultural}

En los siglos XVI y XVII, grandes pensadores económicos percibieron que las actividades artísticas y culturales, a pesar de sus méritos intrínsecos, constituían una desviación del uso del capital, y el trabajo productivo hacia actividades esencialmente improductivas. Para Smith y Ricardo, el arte y la cultura se encuentran en el ámbito natural del trabajo no productivo al no contribuir a la generación de riqueza material de la nación. En sus propias palabras:

Algunos de los trabajos más respetables de la sociedad son como el de los sirvientes: no producen valor alguno que se fije o incorpore en un objeto permanente o mercancía vendible, que perdure una vez realizado el trabajo, [...] En la misma categoría hay que situar a algunas de las profesiones más serias e importantes y también algunas de las más frívolas: [...] actores, bufones, músicos, cantantes de ópera, bailarines, etc. [...] Como la declamación del actor, la arenga del orador y la melodía del músico, la labor de todos ellos perece en el mismo instante de su producción (Smith, [1776] 1994, p. 425-426).

[...] las estatuas y pinturas notables, monedas y libros raros, y los vinos de calidad especial, [...] Su valor no depende en modo alguno de la cantidad de trabajo que ha sido requerida para producirlos, y varía con los cambios en la riqueza e inclinaciones de quienes desean poseerlos. Sin embargo, estos bienes constituyen tan sólo una pequeña parte de todo el conjunto de bienes que diariamente se intercambian en el mercado. Por tanto, al hablar de los bienes, de su valor en cambio y de las leyes que rigen sus precios relativos, siempre hacemos alusión a aquellos bienes que pueden producirse en mayor cantidad, mediante el ejercicio de la actividad humana, y en cuya producción opera la competencia sin restricción alguna (Ricardo [1821] 1959, p. 19-20).

Situación que se explica por el énfasis dado a la teoría del valor trabajo y a la distinción entre trabajo productivo y no productivo, por lo que centraron su atención en "la mano de obra que produce las obras de arte en lugar de las obras de arte mismas" (Goodwin 2006, p. 46), o, como afirma Rausell (1999, p. 86) el problema fundamental es que los bienes artísticos y culturales no encajan en una teoría del valor que no contemple la naturaleza económica del valor estético. No obstante, Smith consideró que las artes ofrecen placer a los individuos y alimentan las posibilidades de pensamiento crítico en la sociedad: 
Si el Estado las estimula, es decir, si garantiza la completa libertad a todos aquellos que por su propio interés procuren sin escándalo ni indecencia entretener y divertir al público con pinturas, poesía, música, baile, con toda clase de representaciones y exhibiciones teatrales, entonces podría conseguir disipar fácilmente en la mayoría del pueblo ese humor melancólico y apagado que casi siempre es el caldo de cultivo de la superstición y el fanatismo (Smith, [1776] 1994, p. 729).

A pesar de lo dicho anteriormente, Smith sostuvo decididamente que el arte podía dejarse a la iniciativa privada, sin participación del Estado, básicamente con dos argumentos (De Marchi; Greene, 2005, p. 439-441): i) el gobierno puede limitar el desarrollo del pensamiento creativo de los individuos a través de la censura; ii) el gobierno no dispone de un mecanismo que haga coincidir sus decisiones con los deseos de los individuos. Además, hay razones ligadas al contexto económico y social de la Inglaterra de Smith que ayudan a entender sus argumentos; las artes estaban en manos privadas y estaban en pleno florecimiento.

Por otra parte, el planteamiento de Bentham, en su famosa analogía con el juego de bolos (push-pin), sugiere que el arte no debe ser distinguido de otras formas de entretenimiento:

La utilidad de todas estas artes y ciencias, me refiero a aquellas de diversión y de curiosidad, el valor que poseen, es exactamente proporcional a la utilidad que ellas proporcionan. [...] Prejuicios aparte, el juego de bolos es de igual valor que las artes y las ciencias de la música y la poesía. Si el juego de bolos proporciona más placer, es más valioso que cualquiera de aquellas. Además, todo el mundo puede jugar a los bolos: la poesía y la música son afición de unos pocos (Bentham, [1843], 1962, chapt. I.: Art and science-divisions).

Sin embargo, Mill (1848, p. 819) abre una importante puerta a la consideración del arte y la cultura como bien de mérito. En efecto, cuando analiza las excepciones a la doctrina, según la cual los individuos son los mejores jueces de su propio interés, encuentra que hay bienes como la educación:

[...] cuyo valor no puede juzgarse por la demanda del mercado, [...] Esto es particularmente cierto en el caso de aquellos bienes que son principalmente útiles porque tienden a elevar el carácter de los seres humanos. Las personas incultas no pueden ser jueces competentes de la cultura.

Posteriormente, Marshall, Keynes y Robbins incorporaron la crítica de los estetas ingleses Ruskin y Carlyle, en el seno de los objetivos de la economía ${ }^{7}$ : no sólo el trabajo que produce riqueza material añade bienestar y riqueza a la economía, también lo hacen la educación y el arte como ingredientes básicos para el progreso de la civilización (Lasuén; García; Zofío, 2005, p. 315-318), aunque,

(7) En la política cultural, este debate es conocido como la "polémica de los estetas" (ver, Palma, 2006). Para los denominados pundits estéticos, el objetivo básico de la humanidad no era el incremento de la riqueza material, como afirmó la economía política clásica y posteriormente Marshall, sino el progreso hacia una mayor civilización, que sólo se podía alcanzar a través de la promoción de las artes y la cultura. 
no consideraron relevante aplicar los instrumentos del análisis económico a las actividades culturales. En palabras de Ruskin ([1862] 2001):

La verdadera ciencia de la economía política, que todavía no se ha distinguido de la ciencia bastarda [...] consiste simplemente en la producción, conservación y distribución, en el momento y lugar más adecuado, de cosas útiles o agradables. [...], y el cantante que con disciplina nunca fuerza su voz [al igual que el granjero, el constructor de barcos y el albañil], son todos economistas políticos en el sentido verdadero y último, contribuyendo continuamente a la riqueza y el bienestar de la nación a la que pertenecen.

En efecto, Marshall ([1890], 2005, p. 261) afirma en los "Principios de Economía" que "[...] el desarrollo de las facultades artísticas del pueblo es en sí mismo un fin de la mayor importancia y se está convirtiendo en un factor principal de la eficiencia industrial":

Así, pues, aunque es cierto que el dinero o poder adquisitivo general, [...], es el centro alrededor del cual gira toda la ciencia económica, esto es así, $[\ldots]$ porque en este mundo es el único medio apropiado para medir los móviles humanos en gran escala. Si los antiguos economistas hubieran aclarado este concepto, habrían evitado muchas malas interpretaciones, y las espléndidas enseñanzas de Carlyle y de Ruskin acerca de los rectos propósitos del esfuerzo humano no habrían sido oscurecidas por los rudos ataques dirigidos a los economistas, ataques fundados en la falsa creencia de que aquella ciencia no se ocupaba de más móviles que el deseo egoísta de riquezas y de que inculcaba una política de sórdido egoísmo.

Keynes ([1930], 1963, p. 362), en "Economic Possibilities for our Grandchildren", hace una llamada de atención al hecho de que el hombre, una vez liberado de las "presiones económicas", debe enfrentar su verdadero problema: "¿cómo ocupar el ocio, que la ciencia y el interés compuesto han ganado para él, para vivir con sabiduría y agradablemente?", situación en la que el arte y la cultura ocupan un lugar central. En el mismo sentido, a través del Bloomsbury Group, insistió en que el arte era un componente básico y esencial para el logro de la verdadera civilización humana (Moggridge, 2005, p. 540; Goodwin et al. 2006, p. $65)$ :

- La experiencia estética derivada del consumo de los bienes y servicios artísticos es diferente frente al consumo de otros bienes y servicios.

- En los mercados de arte, el precio no es el único impulsor de la creación artística. En éstos, pareciera influir más una presión psicológica interna en la producción de las obras que la recompensa financiera para producirlas.

- La educación artística es tan importante como la formación técnica, normalmente la educación técnica se justifica porque puede producir un aumento en la producción de bienes y servicios a partir de un conjunto de recursos. Igualmente, la educación en las artes y la literatura se justifica por su capacidad para permitir a los seres humanos vivir una vida mejor.

Por último, Robbins, desde sus escritos "Art and the state" (1963) y "Unsettled Questions in the Political Economy of the Arts" (1971), fue defensor 
del apoyo con fondos públicos y privados a las artes. Dos fueron sus argumentos: i) la educación debe incluir desde edades tempranas el contacto con las artes, dado que éstas contribuyen a hacer más placentera la vida $\mathrm{y}$, ii) la disponibilidad de lugares y servicios que ofrezcan un amplio acceso a las artes (museos, bibliotecas, teatros) que garanticen el conocimiento y preservación de la herencia cultural a lo largo del tiempo. En particular, a la pregunta ¿por qué el contribuyente debe proporcionar dinero a las artes? Robbins advierte que esta es una cuestión de filosofía política asociada a la función del Estado y al sentido que las artes le imprimen a la sociedad:

¿Por qué el contribuyente debería proporcionar dinero para apoyar a las artes? ¿Por qué no todo el negocio se deja a la demanda de los consumidores? Si la gente desea arte lo comprará: sino, ¿por qué debe ser producido? [...] ciertamente esta no es una pregunta que deba responderse haciendo referencia a la economía científica. Es un asunto de los más altos valores, un asunto de lo que se piensa debe ser el propósito y la función del Estado como elemento autoritario en la sociedad, un asunto de filosofía política. A la economía se recurre sólo cuando se quieren conocer las implicaciones de nuestras decisiones a este respecto [...] (Robbins, 1971, p. 67).

En este breve repaso a economistas centrales en la historia del pensamiento económico, se encuentran los principales argumentos que constituyen el centro de debate en la polémica sobre la financiación pública del arte y la cultura (Goodwin, 2006): De un lado, el planteamiento de que el arte y la cultura, a pesar de reconocerle su importancia para la sociedad, no presenta características excepcionales que la diferencien de otros bienes. Por tanto el arte y la cultura pueden existir sin el apoyo del gobierno, como cualquier otro bien en el mercado. De otro lado, el planteamiento de que el arte y la cultura son ingredientes clave para la vida en sociedad y contribuyen a dar calidad y sentido a la vida, en este contexto la experiencia estética derivada del consumo cultural genera externalidades positivas. En efecto, muchos de los bienes culturales presentan características de bien público $\mathrm{y} / \mathrm{o}$, desde una perspectiva de las preferencias comunitarias, se pueden catalogar como bienes de mérito, lo que en ambos casos implica la intervención del Estado.

\subsection{Los primeros argumentos a favor del apoyo con fondos públicos al arte y sus críticas}

La intervención del Estado en las actividades artísticas y culturales y, en concreto, en la financiación se justifica en función de que el mercado falla en su producción y distribución y/o de que el producto de estas actividades constituya un bien de mérito (Cwi, 1979). Se entiende que se produce un fallo del mercado, cuando por alguna razón, entre ellas, el precio de un bien, las preferencias de los individuos, la distribución de la renta, la disponibilidad de información, o alguna combinación de éstas y otros factores, implica una producción inferior a la 
deseada, se impide el consumo de algún sector de la población o se limita la variedad $\mathrm{y} / \mathrm{o}$ la calidad del producto.

Desde la obra seminal "Performing Arts: The Economic Dilemma" de Baumol y Bowen (1966), se advierte el reto que significa la financiación de la cultura al interior de la política cultural. El "dilema económico" configuró la llamada "enfermedad de los costes", propia de las artes escénicas, situación que llevaba consigo una clara prescripción de política económica, el Estado debe subsidiar las artes escénicas (teatro, opera, danza, música clásica).

¿Cuál es el "dilema" que observaron Baumol y Bowen? Veamos, producto de una investigación estadística, con información de la evolución de los costes de operación por concierto para la "Orquesta Filarmónica de Nueva York" entre 1843 y 1964 como también, una comparación del coste medio por presentación del Drury Lane Theatre en las temporadas 1771-72 y 1775-76 con el Royal Shakespeare Theatre en la temporada 1963-1964, los autores encuentran (Baumol; Bowen, 1965, p. 499-500; 1966, p. 183-186; Baumol, 1967a, p. 47-48):

Primero, que los salarios de los artistas e intérpretes aumentan más lentamente que los salarios de la economía en general.

Segundo, que la mayor parte de los costes totales (entre $70 \%$ y $80 \%$ ) en las artes escénicas lo representan los salarios.

Tercero, que los costes totales de las artes escénicas presentan un aumento constante a través del tiempo y por encima de los costes de producción de la economía en su conjunto.

El dilema, ¿por qué, si los costes en las artes escénicas se componen en su mayoría de salarios, y si éstos aumentan más lentamente que los de otros sectores en la economía, los costes de producción en las artes escénicas aumentan más rápidamente que los de la economía en su conjunto?

La explicación del dilema es una consecuencia de las características técnicas de la producción de artes escénicas (Baumol, 1967b). Consideremos un modelo de dos sectores: uno de alta productividad que admite cambios tecnológicos rápidos que ahorran mano de obra (manufacturas; sector progresivo) $\mathrm{y}$ uno de baja productividad, intensivo en trabajo, que admite mejoras tecnológicas lentas y esporádicas (artes escénicas; sector no progresivo). A lo largo del tiempo, los salarios se igualan entre sectores y crecen a la misma tasa que la productividad del trabajo en el sector de alta productividad; esta situación genera dos efectos: el coste relativo de los bienes del sector de baja productividad crece en forma continua; el incremento del precio relativo de los bienes del sector de baja productividad, si su demanda no es inelástica al precio o no es elástica al ingreso, lleva a que los niveles de producción de dicho sector se reduzcan e incluso desaparezcan.

La Figura 1 ilustra la "enfermedad de los costes" para la producción de un hipotético bien cultural (artes escénicas). En la situación inicial, reflejada por la 
curva de demanda $(D)$ y la curva de oferta $(O)$, se produce la cantidad $Q_{*}$ al precio $P *$ Si la brecha tecnológica, es decir, la dificultad de absorber rápidamente el cambio tecnológico, implica que el coste de producir el bien del sector estancado aumente, la oferta se desplaza a $O_{l}$ y se produce ahora $Q_{l}$ a $P_{l}$. Comparando el gasto inicial $P_{*}^{*} Q_{*}=A+C$ con el final $P_{I} * Q_{l}=A+B$, se observa que ahora se está produciendo una menor cantidad del bien del sector estancado y el gasto requerido es mayor $(B>C){ }^{8}$ Esta situación implica que, para sostener los mismos niveles de producción del bien cultural, se requiere un mayor esfuerzo financiero de los consumidores, del Estado o del mecenazgo para cubrir la diferencia entre $\mathrm{P}_{1}$ y $\mathrm{P}_{*}$. En palabras de Baumol y Bowen (1965, p. 502):

Si nuestro modelo es válido y, si como se puede sospechar, hay límites a las cantidades que se pueden obtener de los contribuyentes privados, habrá que encontrar un mayor apoyo de otras fuentes, para que las artes escénicas puedan continuar con el papel que desempeñan en la vida cultural de este país y sobre todo si se tiene la intención de ampliar el rol que desempeñan en la sociedad y prosperen rápidamente.

Figura 1

Una ilustración gráfica de la "enfermedad de los costes"

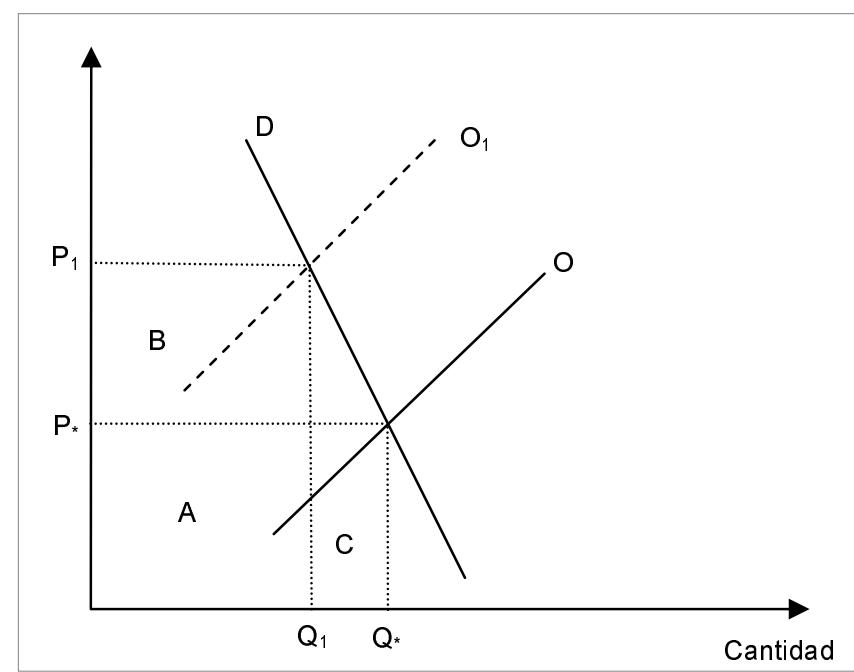

Fuente: Elaboración propia.

(8) La "enfermedad de Baumol" se extendió rápidamente a los servicios en general y a sus efectos sobre el crecimiento económico. En efecto, Baumol observa que hay una producción del sector estancado que corresponde a servicios altamente inelásticos al precio, o elásticos a la renta, o que el gobierno decide mantenerlos a través de subsidios, p. ej., la educación y la salud. Además, la tasa de crecimiento agregado de la productividad es un promedio ponderado de las tasas de crecimiento sectorial, dado los pesos proporcionados por el valor nominal de cada sector en total del PIB. En este contexto, la tasa de crecimiento de la productividad global se reducirá con el tiempo, dado que el sector estancado recibe un peso cada vez mayor. Por tanto, como consecuencia de un crecimiento desequilibrado, la tasa de crecimiento del PIB de las economías tiende a estancarse, ver al respecto Nordhaus (2008). 
Al argumento económico de la "enfermedad de los costes", se suma el argumento desde la crítica a la economía política clásica realizada por los estetas ingleses. Argumento que, desde la economía, interpreta al arte y la cultura como bien de mérito que, si bien resulta antiguo desde el pensamiento económico el concepto como tal, data desde finales de la década de 1950 en la teoría económica. En general, Musgrave ([1959] 1968) encuentra que con el producto de la interacción social, la simpatía y los valores comunitarios, los individuos llegan a compartir preocupaciones hacia ciertos valores y bienes que aceptan sustituir la norma de soberanía del consumidor por otro tipo de norma asociada a "[...] una diferencia de actitudes psicológicas y de filosofía social respecto a los [...] tipos de bienes" (Musgrave, [1959] 1968, p. 68).

En particular, el caso de los bienes de mérito implica la existencia de necesidades preferentes por parte de la sociedad. Por diversas razones, los individuos no evalúan correctamente los beneficios que derivan del consumo de una clase particular de bienes, lo que conduce a que se produzca una cantidad subóptima de éstos. Esta última situación conlleva a la intervención del Estado, que se justifica en el sentido de corregir las preferencias individuales y alcanzar una adecuada provisión del bien. ${ }^{9}$

Es importante diferenciar entre bien público y bien de mérito. En un bien público puro, resultan clave dos aspectos técnicos derivados de las características de los bienes (la no rivalidad y la no exclusión) que afectan al incentivo para su provisión vía mercado, generando un problema de oferta (Samuelson, 1955). En el caso de los bienes de mérito, el problema es de demanda. Se hace necesario potenciar el conocimiento de este tipo de bienes a los individuos, por ello su consumo se considera que debe ser obligatorio. Igualmente es importante no confundir, como usualmente se realiza, a los bienes públicos con los bienes que suministra el Estado. Como señala Sandmo (2008), el adjetivo "público" y/o "privado" no prejuzga o indica nada al respecto de las actuaciones del sector público o privado hacia estos bienes. Su definición corresponde a criterios tecnológicos y no institucionales. Algunos de los bienes culturales presentan características de bienes públicos o cuasi-públicos, dadas las condiciones de no rivalidad en el consumo y oferta no exclusiva (una muralla histórica, la fachada de una catedral, un festival al aire libre, un museo ${ }^{10}$ ).

(9) No existe una única definición de bien de mérito, es más, ha habido una gran polémica acerca de su definición, alcance y uso dentro de la teoría económica (ver, Mazzanti, 2002, p. 531-536).

(10) Bajo el supuesto de que el coste de entrada (generalmente muy bajo y subsidiado) no represente una restricción en las condiciones de acceso y disponibilidad del museo. Y, además, suponiendo de que no se presente congestión, que implicaría la presencia de rivalidad en el consumo. En general muchos estudios consideran a los museos bienes públicos (Herrero; Sanz; Bedate, 2003, p. 30-31). Para un análisis económico del funcionamiento de un Museo ver, Frey y Meier (2006). 
La "enfermedad de los costes" y el concepto bien de mérito constituyen, desde mediados de 1960, el principal apoyo para justificar la financiación con fondos públicos del arte y la cultura. En efecto, cuando en 1965 se crea en EE.UU. el National Endowment for the Arts, sus promotores en el congreso se centraron en dos argumentos (Moen, 1997, p. 186): i) que las artes deben estar disponibles para toda la gente en una "gran sociedad", y ii) que las artes se deben valorar igual que las ciencias y la tecnología.

Las críticas a los primeros argumentos no se hicieron esperar. Peacock (1969) sostiene que, a pesar del "contagio" de la "enfermedad de los costes" por parte de las artes escénicas, ésta no constituye un argumento que justifique su financiación por parte del Estado. Lo que sí es claro es que la supervivencia de aquellas dependería de la existencia de fuentes de financiación distintas de los ingresos directos por venta de entradas. En este sentido, sugiere que el principal argumento para la acción pública a favor de las artes escénicas es la satisfacción derivada por las generaciones presentes de conservar y legar a las generaciones futuras las artes escénicas.

Para ello, Peacock (1969, p. 159) señala dos acciones a ejecutar por parte del gobierno: i) a corto plazo, desplazar el subsidio otorgado a las instituciones que promueven y producen el arte hacia los consumidores y, ii) a largo plazo, encontrar un mecanismo que permita alterar las funciones de preferencia de las generaciones futuras para contrarrestar la "enfermedad de los costes" a través del mercado. En el primer caso, recomienda como herramienta introducir el sistema de vales, $\mathrm{y}$, en el segundo caso, redirigir los aumentos de los fondos públicos para el apoyo al arte a través del sistema educativo. La formación artística permite una mayor apreciación y valoración del arte por parte de los individuos y a su vez puede incidir en el surgimiento de nuevos artistas.

Scitovsky (1972) encuentra que la asistencia promedio a espectáculos de artes escénicas en EE.UU., es la mitad de la que exhiben algunos países de Europa, a pesar de que los adelantos tecnológicos producidos en EE.UU. liberan tiempo y energía a la población para perseguir una "vida interesante y agradable". En su opinión, el problema que refleja la "enfermedad de los costes" sobre la financiación de las artes escénicas no es un problema de la economía, sino más bien de la escasa educación en las artes de la población que no permite que ésta disponga de las habilidades necesarias para entender, apreciar y disfrutar de las artes.

De acuerdo con Scitovsky (1972, p. 68), ninguno de los argumentos usuales a favor de la financiación pública es realmente aplicable a las artes y la cultura: i) sus características particulares como bienes o servicios no hacen que, por razones de equidad o eficiencia, deban ser proporcionados por el gobierno; 
ii) su subsidio no es una herramienta adecuada para mitigar las desigualdades de renta y promover la asistencia, ni puede estar justificado por razones de protección de los intereses de las generaciones futuras. Así, el único argumento válido para la intervención pública en las artes es la educación de la población en las artes que le permita disfrutar y enriquecer su vida a través de ellas.

En el mismo sentido, con respecto a la "enfermedad de los costes" Cowen y Grier (1996) encuentran que, por el contrario, el crecimiento económico tiene efectos favorables sobre la producción artística, en particular en bienes de alta elasticidad ingreso como las artes escénicas. Heilbrun y Gray (2001) indican que la dificultad de absorber rápidamente el cambio tecnológico, en las artes escénicas, no constituye un argumento válido para justificar un subsidio que no refleje los verdaderos costes que enfrenta la industria. Y, en general, los estudios empíricos si bien muestran que los costes han crecido en las artes escénicas como predice el modelo, los cambios tecnológicos (nuevas tecnologías de grabación y distribución) mitigan la relación trabajo-intensiva propia de estas actividades (Throsby, 1996; Frey, 1996; Heilbrun, 2003). Adicionalmente a la introducción de técnicas de mercadotecnia que generan fuentes de ingresos distintas de la venta de entradas (Throsby, 2008). ${ }^{11}$

En general, las principales críticas a las ayudas directas (subsidios) a las actividades culturales se han dirigido a poner en duda: i) sus efectos distributivos, ii) las externalidades generadas por el arte y la cultura y, iii) su consideración como bien de mérito. De acuerdo con Van den Haag (1979):

Primero. No hay ninguna buena razón socio-política para que el gobierno obligue a los contribuyentes a subvencionar el arte, los beneficios externos que rinde el arte y la cultura no han sido demostrados plenamente, al menos para EE.UU.:

Sin importar el valor de la ópera (y yo soy un apasionado amante de la ópera) no puede decirse, como puede hacerse en Italia o Austria, que la ópera haya contribuido a la cohesión nacional, histórica, cultural o de conciencia - o que tiene oportunidad de hacerlo ahora. [...] Lo que es cierto para la ópera es cierto para la música clásica, para la danza, incluyendo al ballet, y, en general, para las grandes obras de arte en nuestros museos. Ellas no desempeñaron un papel importante en nuestra historia o en la creación y forja de nuestros vínculos nacionales [...] (Van den Haag, 1979, p. 66).

Segundo. Lo que efectivamente hace el gobierno es obligar a todas las clases a subsidiar a la clase media y alta que son, efectivamente, quienes asisten a

(11) Para ampliar los anteriores argumentos, se pueden consultar los artículos que aparecen en el número monográfico del Journal of Cultural Economics, v. 20, n. 3, 1996, dedicado al "The 30th Anniversary of The Performing Arts: An Economic Dilemma by Baumol and Bowen". 
las artes escénicas, ${ }^{12}$ en especial a la ópera. Así, los subsidios del gobierno interfieren con la libertad de los individuos para gastar de acuerdo con sus preferencias, por ejemplo "en una película de cine no subsidiada".

Tercero. La proliferación de programas de gasto público dirigido a subvencionar el arte es probable que haga más daño que bien a la creación de arte de calidad. El gobierno no dispone de un mecanismo que le permita determinar qué arte es "bueno" y qué arte es "malo", de forma que al otorgar subsidios indiscriminadamente puede atraer a "seudo-artistas" motivados por el ingreso que pueden obtener del presupuesto público.

En la misma línea, Banfield (1984) sostiene que la financiación pública del arte y la cultura, basada en el argumento de que aquellos generan externalidades positivas, es sumamente débil, pues dichas externalidades no han sido demostradas. Y, los subsidios a las artes, como, por ejemplo, las entradas de los museos, no se deben otorgar, más bien se deben producir políticas generales, que mejoren la distribución de la renta y no subvencionar precios. Igualmente, en una de las ya clásicas polémicas sobre la financiación de las artes entre West $(1986)^{13}$ y Grampp (1986/1987), este último se pregunta si; ¿deberían financiarse a sí mismas las artes? La respuesta en su opinión es sencilla; “[...] el arte sigue siendo un bien privado. La gente que lo desea debe pagar él"' (Grampp, 1986/1987, p. 43).

Al argumento sobre si el arte es un bien de mérito, la posición de Frey (2000, p.127) refleja el punto de vista crítico:

Desde el punto de vista económico, este concepto no es convincente por dos razones: i) Es difícil justificar por qué en un mundo en el que se da por sentada la soberanía del consumidor puede concebirse la existencia de bienes sobre cuya utilidad decide otro que no sea el consumidor, ii) El arte y la cultura valdrían por definición tanto como la cantidad de dinero que los políticos estuvieran dispuestos a invertir en su ayuda, lo que no tiene mucha utilidad para guiarnos en la práctica.

Grampp (1989a), en el capítulo 7 de su libro "Pricing the Priceless: Art, Artists, and Economics", resume los principales argumentos empleados por los economistas para sustentar la ayuda pública a las artes. Tanto aquellos que se relacionan con la demanda, es decir, "sobre los beneficios que el arte rinde a quien

(12) El trabajo seminal de Baumol y Bowen (1966) encontró que los asistentes a las artes escénicas en Reino Unido y EE.UU. eran con respecto a la población general de mayores ingresos, mejor educados y blancos.

(13) La propuesta de West, que motivó a la réplica de Grammp, fue la de emplear "vales" o "cupones" que permitan la entrada a los escenarios o representaciones con un coste menor, para devolver el poder de decisión a los consumidores sobre a qué función o actividad cultural asistir y, por tanto, a cuál apoyar con los recursos públicos, considerando que este poder ha estado en manos de los administradores de las instituciones artísticas y culturales que reciben la financiación directa ya sea del Estado, la empresa privada o los particulares. Esta situación puede implicar, a través del lobby, la captura de rentas por parte de los "administradores del arte" que sin consultar las preferencias de los agentes deciden que arte finalmente llega al público. 
goza de él", como aquellos que se relacionan con alguna característica de la oferta, es decir, "en la manera que se crea el arte y lo que cuesta". ${ }^{14}$ En su concepto, los argumentos son "poco convincentes" y no demuestran que, efectivamente, las artes sean merecedoras de algún tipo de subvención por parte del presupuesto público, incluso del sector privado. Para que fuera así, al menos, debe demostrarse que se cumplen dos condiciones: que individualmente son necesarias y en conjunto suficientes: i) que el coste de oportunidad de la subvención sea el más elevado entre las múltiples alternativas que tenga a su alcance el donante, ya sea el gobierno, una empresa privada o un particular, ii) en el caso de fondos públicos, el dinero de la subvención debe provenir de impuestos que paguen los beneficiarios de la actividad que se financia, tal como hace la gente con lo que recibe del sector privado. ${ }^{15}$ En resumen, ninguno de los argumentos cumple estas condiciones.

Asimismo, Grampp (1989b), en su artículo "Rent-seeking in arts policy", expone, a su juicio, la principal razón por la cual la ayuda del gobierno a las artes es una realidad. La respuesta que ofrece indica que la forma organizativa de las instituciones que administran el arte (en general, organizaciones sin ánimo de lucro ${ }^{16}$ ) y sus integrantes (los artistas, los administradores y los que disfrutan el arte) se prestan para la búsqueda de rentas:

La asistencia gubernamental a las artes es una transferencia involuntaria de recursos del público a las organizaciones de arte y su público. La transferencia no es resistida porque el costo para las personas que pierden es pequeño y son numerosos, mientras que los beneficiarios son pocos y sus beneficios son considerables. [...]. Que las rentas son, de hecho, solicitadas y obtenidas es evidente en el comportamiento de las organizaciones que administran el arte. Que ellas y su público son los principales beneficiarios es evidente al examinar la forma en que la asistencia se da (Grampp, 1989b, p. 120).

Frente a las fuertes críticas recibidas por los primeros argumentos y en la búsqueda de un argumento "objetivo" para justificar la financiación pública del arte y la cultura en la década de 1970, se inician los "Estudios de Impacto Económico de la Cultura (EIEC)", que explicamos a continuación.

(14) Argumentos por el lado de la demanda: i) el arte como bien público, ii) el arte genera externalidades positivas, iii) el arte es un bien de mérito, iv) la demanda de arte depende de la oferta, v) el arte debe estar a disposición de todo el mundo. Argumentos por el lado de la oferta: vi) el arte se crea en condiciones de coste medio decreciente, vii) el sostenimiento de las existencias de arte, y viii) el arte sufre de la "enfermedad de los costes".

(15) "Esta condición no se aplica para una subvención con el propósito de redistribuir el arte por razones de igualdad" (Grampp, 1989a, p. 249).

(16) Las empresas sin ánimo de lucro son muy usuales en el sector cultural, para un análisis que explica esta situación ver Netzer (2003). 


\subsection{Los estudios de impacto económico empleados como argumento para justificar el apoyo con fondos públicos al arte y la cultura}

Los "Estudios de Impacto Económico de la Cultura (en adelante EIEC)", dentro de un marco keynesiano, buscan determinar el flujo de gasto-renta que genera una actividad cultural en una región determinada y la interrelación que estos flujos producen sobre la actividad económica general vía multiplicadores (Throsby, 2004). El objetivo de estos estudios, en gran parte, fue convencer a la opinión pública y a los policy makers, de que el sector cultural, dada su contribución al PIB y al empleo, era merecedor de la financiación pública. ${ }^{17}$

Sin embargo, el argumento resultó muy débil. Madden (2001, p. 165-166) encuentra que asociar el tamaño o la importancia de un sector, en términos de su aportación al PIB total de la economía, con la financiación pública, puede resultar ingenuo. No existe, ni teórica, ni empíricamente, un determinado nivel de importancia de un sector que justifique su apoyo económico con recursos públicos:

Considere una analogía médica: la piel es el órgano más grande del cuerpo, ¿pero es el más importante? Sin distraerse en temas de morbilidad o de mortalidad, es más útil ver el cuerpo como un todo integrado, como una colección de fenómenos médicos cuya importancia se mide por el papel que desempeñan en la unidad hacia la homeostasis. Pequeños órganos pueden ser tan importantes como los grandes órganos. Las industrias en la economía son análogas.

En este contexto, no existe un punto de referencia adecuado para la comparación del peso de la cultura versus otra u otras industrias o servicios, para medir su importancia. Por ejemplo, no tiene sentido comparar una galería de arte, con un casino, o con una guardería, o con un centro para ancianos, o con la industria de la construcción o de producción de armas, sin considerar sus valores intrínsecos, la finalidad de la actividad y las externalidades que generan. Incluso, el uso de los EIEC para justificar el apoyo con fondos públicos, puede generar efectos contraproducentes al invitar al gobierno sólo a apoyar aquella parte de las actividades culturales que generan efectos económicos medibles (Madden, 2001, p. 172).

Asimismo, los estudios de impacto económico aplicados a cualquier actividad presentan limitaciones con respecto a: i) la precisión geográfica de la unidad de análisis, aspecto necesario para estimar los costes de oportunidad de la

(17) Una situación que contribuyó a la visibilidad económica del sector cultural en la década de 1970 fue la ampliación del espectro de los bienes culturales: desde la noción restringida de "alta cultura" vinculada a las artes escénicas, a la inclusión de la "cultura popular" (patrimonio, música popular, festivales) y a las industrias culturales (cine, literatura, artesanía) (Peacock, 2000; Throsby, 2008). Situación que a su vez generalizó el uso del término cultura por el de arte en la denominación de la subdisciplina (Towse, 2003) por considerarse más amplio y menos restrictivo que el de arte.

Economia e Sociedade, Campinas, v. 20, n. 1 (41), p. 195-228, abr. 2011. 
tierra y otros recursos que potencialmente se pueden emplear en otras actividades o proyectos, ii) el supuesto de que en ausencia de una actividad o proyecto determinado los gastos asociados no se hubiesen generado, omitiendo las filtraciones que usualmente se presentan en los flujos de ingresos y gastos de las economías y, iii) el no uso de multiplicadores específicos de las actividades, lo que conlleva a sobrevalorar los impactos indirectos (Johnson; Sack, 1996, p. 374).

El mayor número de EIEC se ha realizado para el caso de los festivales de arte; al respecto Snowball y Antrobus (2002) encuentran que frecuentemente en estos estudios se presentan los siguientes errores:

Definición del área de estudio, los resultados del impacto económico son sensibles a la determinación del tamaño del área geográfica donde se realiza el evento o actividad. En general, a mayor área geográfica menores serán las filtraciones de ingresos y gastos $\mathrm{y}$, por tanto, mayor el multiplicador y el impacto económico.

Inclusión de los espectadores y/o visitantes, una vez definida el área geográfica del evento o actividad, en principio, sólo los asistentes de fuera de esa área deben incluirse en el análisis, dado que su gasto representa una nueva inyección de ingreso al área. Generalmente, la separación entre asistentes que residen fuera del área del evento y los "residentes" en el área se realiza a través de preguntas en el momento de la compra de entradas y por las reservas de alojamiento. ${ }^{18}$

El caso de visitantes casuales, puede presentarse el caso de visitantes que se encuentren en la zona por razones diferentes (visita a familiares, cerrar un negocio, escala de avión, etc.) a la presencia de la actividad o proyecto en cuestión, y deciden asistir a las actividades programadas para ocupar parte de su tiempo. Por tanto, los gastos de estos visitantes causales no se explican o se asocian a la presencia del evento o actividad particular objeto de análisis $\mathrm{y}$, por ello, no se deben contabilizar como impacto generado consecuencia de las actividades programadas.

El multiplicador adecuado, el cálculo de un multiplicador específico para la actividad requiere de un proceso de recolección de información primaria y secundaria bastante dispendioso en tiempo y recursos. Esta situación hace que, generalmente, se recurra a multiplicadores empleados en otros sectores o en zonas similares, lo que resulta en cálculos que no necesariamente reflejan la realidad de la actividad particular que se está analizando.

(18) En algunos estudios se refina el cálculo, en el sentido de tener presente que algunos residentes en el área no se desplazaron a otros lugares por la presencia del evento o actividad. Por tanto, sin la presencia de la actividad se habrían fugado estos ingresos. Algo así como una equivalencia entre hogares que deciden quedarse de vacaciones en su casa y asistir a los eventos programados en lugar de desplazarse de vacaciones a otras zonas, producto de una elección racional. 
Inclusión de todos los costes de oportunidad de los recursos empleados, es importante tener presente el coste de oportunidad de todos los recursos tanto públicos como privados invertidos en la actividad. Por ejemplo, el gasto de los gobiernos locales o regionales en la financiación de un evento no puede ser considerado como una nueva inyección de recursos en el área donde se realiza la actividad, puesto que constituyen una transferencia de los habitantes de la zona en forma de impuestos, frente a otros usos potenciales de estos recursos. Igualmente, se deben contabilizar como costes el gasto local adicional de presencia policial, de recolección de residuos (basura), las molestias asociadas al aumento de la delincuencia, el incremento del tráfico, el ruido y el gasto de residentes de la zona que debido a las actividades deciden desplazarse a otros lugares.

Seaman (2003, p. 229) agrega el "error de interpretación de las políticas" (el equilibrio general frente al equilibrio parcial) entendido como la presunción incorrecta de que un impacto económico positivo es condición suficiente para solicitar financiación del gobierno, sin considerar los costes de oportunidad de desviar dicha contribución de otras inversiones del sector público que, potencialmente, podrían alcanzar una tasa de rentabilidad más alta. Por su parte, Devesa (2006, p. 159) encuentra que en general estos estudios son ex-post y no exante, lo que los haría más útiles.

En resumen, como ha señalado Seaman (1987, p. 746), aquellos que proponen los EIEC como argumentos para la financiación estatal de la cultura están eligiendo jugar con las cartas más débiles, en lugar de jugar con los ases: las externalidades positivas generadas por las artes a la sociedad, principal causa de fallos de mercado, que deberían emplearse para motivar el apoyo público. No obstante, como lo reconoce Throsby (2004, p.25): "La tarea de cuantificar la amplitud de los beneficios como bien público del arte, si es que existen, ha resultado más difícil”. En la misma línea, Frey (2000, p.175) afirma que:

[...] la forma en la que estos estudios de "Impacto Económico" miden el ingreso monetario no es correcta porque no calculan el excedente social neto, es decir, no deducen los costes del total de la producción (los pagos de salarios no son un beneficio sino un coste). La industria cultural es muy aficionada a pedir este tipo de estudio, pese a que confunde la mera utilización de recursos con un beneficio social. Sin embargo, no tiene en cuenta los valores que no se reflejan en el mercado. [...] el patrimonio cultural y la actividad artística, en general, tienen valores de "existencia", "prestigio", "opción", "educación" y "legado", cuyo monto hay que calcular con la mayor precisión posible.

Esta situación se explica desde la diferente concepción de valor que se encuentra; entre aquellas personas involucradas en el mundo de la cultura (administradores, empresarios, artistas), la "gente de la cultura" y, los profesionales de la economía que estudian los aspectos culturales, los "economistas de la cultura" (Frey, 2008, p. 262-263): 
La "gente de la cultura", toma el valor de la cultura como algo ya establecido, que no es necesario demostrar que contribuye al bienestar humano, por tanto consideran inherente al Estado su financiación. Por ello, prefieren los estudios de impacto, de forma, que se cuantifiquen los efectos económicos (gastos en proveedores, número de empleos generados) de una actividad cultural concreta, como puede ser la correspondiente a un museo o a un festival. De esta forma, se justifica la movilización de recursos públicos para su financiación.

Los "economistas de la cultura", consideran que es esencial demostrar la necesidad del apoyo del Estado a cualquier proyecto cultural, para ello, se requiere la presencia de efectos externos positivos que justifiquen dicho apoyo. En este sentido, prefieren los estudios de la disposición a pagar (a través de la valoración contingente) con los que se miden los efectos externos, es decir, los efectos de las actividades culturales relacionados con el aumento del bienestar que no es recogido por el mercado.

En efecto, el valor económico de un bien particular puede ser definido y estimado con apreciable certeza. Un templo histórico, por ejemplo, puede tener un precio de venta, como activo real que es, y un valor de no mercado cuantificado por la disposición a pagar de las personas por su conservación. No obstante, el valor económico no necesariamente recoge la complejidad del valor cultural del activo como puede ser: la calidad estética, significado espiritual, importancia simbólica o histórica, influencia en el desarrollo de estilos arquitectónicos (Throsby 1999; 2001).

Desde una perspectiva sociológica se critica el excesivo énfasis en los "beneficios instrumentales" [económicos] del arte y de la cultura: "estos argumentos se han apropiado ampliamente de la terminología económica [...], concentrándose en la medición cuantitativa de los costes y beneficios y la demostración empírica de los efectos como razón fundamental para el apoyo a las artes" (McCarthy; Ondaatje; Zakaras; Brooks, 2004, p. 67). Perdiendo de vista los "beneficios intrínsecos" que producen las actividades artísticas y culturales asociados al "enriquecimiento de la vida de la gente", a través del placer y la estimulación emocional, que son beneficios que constituyen la principal razón que explica la participación de las personas en estas actividades. Además, los "beneficios intrínsecos" generan efectos positivos para toda la sociedad asociados a un incremento de la capacidad de los individuos para percibir, sentir e interpretar el mundo: mayor capacidad de empatía y de creación de lazos sociales, NESF (2007, p. 4-11).

DiMaggio (2002) identifica tres falacias contenidas en el discurso de la política cultural sobre los beneficios del arte y la cultura. En particular, el autor advierte sobre los peligros de suponer que: i) todas las formas de participación en las artes son iguales (falacia de homogeneidad de tratamiento); ii) la exposición a las artes genera el mismo efecto sobre los diferentes tipos de participantes y en 
diferentes tipos de comunidades (falacia de homogeneidad de efecto); iii) los beneficios aumentan como una función lineal de la participación en las artes (falacia de linealidad de efectos). En general, DiMaggio no duda sobre la existencia de los beneficios positivos de las artes sobre la comunidad, pero advierte que éstos dependen de la naturaleza específica de cada actividad y de las circunstancias particulares de la comunidad en que se desarrollen.

En este sentido y motivados por las críticas a los EIEC, ya que es uno de los principales interrogantes que enfrenta la subdisciplina de la economía de la cultura; ¿cómo valorar las externalidades que el consumo cultural genera? desde una perspectiva microeconómica, se introducen metodologías para valorar las preferencias individuales por los bienes culturales bajo condiciones hipotéticas (contingentes) $\mathrm{y}$, de esta forma, obtener valoraciones socialmente deseables en función de la contribución de los bienes culturales al bienestar de la sociedad.

\subsection{Cuantificando el valor cultural}

Dado de que algunas características de los bienes culturales no se valoran a través del mercado (por ejemplo, el valor arquitectónico e histórico de una catedral), se emplean metodologías que buscan determinar la "disposición a pagar" de los consumidores por un bien o servicio. La disposición a pagar de los demandantes expresa: "el precio máximo que éstos estarían dispuestos a pagar por consumir un bien". Precio que se refleja en los denominados: valor de uso y valor de no uso. El primero está asociado al excedente del consumidor, es decir, la estimación de la utilidad o del beneficio que proviene del consumo directo del propio bien o de los servicios que se derivan del mismo. El segundo representa aquellos valores generados por el bien cultural no fácilmente identificables a través de las transacciones de mercado reflejadas en los precios (valores de existencia, opción y legado).

Una aproximación a la medición de estos tipos de valores puede hacerse desde metodologías que trabajan con dos enfoques (Herrero et al., 2003, p. 42-45; Mourato; Mazzanti, 2002, p. 53-57): i) preferencias reveladas, a través de mercados sustitutos, como los precios hedónicos (demanda de bienes similares) o los costes de viaje (demanda de bienes complementarios), y ii) preferencias declaradas, a través de mercados o situaciones hipotéticas, como la Metodología de la Valoración Contingente (en adelante, MVC) y los Choice Experiments (en adelante, $C E)$.

La $M V C$ constituye la metodología más empleada en la valoración de bienes culturales desde el primer lustro de los años ochenta del siglo pasado 
(Cuccia, 2003, p. 124). ${ }^{19}$ La idea de la $M V C$ es valorar un bien en un mercado hipotético, a través de una entrevista a una muestra estadísticamente significativa y seleccionada de personas, que indaga acerca de la voluntad o intención de pagar por ese bien. Es decir, se simulan las transacciones que, potencialmente, se producirían en la realidad en el mercado del bien y de esta forma se estima su valoración en términos monetarios.

Frey (2000, p.185-186) encuentra tres aspectos positivos en el desarrollo de estudios de valoración contingente aplicados a los bienes culturales:

i) En el esfuerzo de investigación que requiere el objeto artístico a evaluar, el cuestionario debe cumplir con estrictos requisitos para poder emplearse y, además, ser específico para el bien en cuestión y, aplicarse tanto a potenciales visitantes como potenciales no visitantes.

ii) La $M V C$ permite capturar y estimar los valores de existencia, opción y legado, lo que significa aproximarse a la valoración monetaria de los valores de no uso, que alcanzan notable importancia en las actividades culturales.

iii) La $M V C$ igualmente permite aproximarse a la evaluación y valoración de la calidad de los servicios prestados por el bien cultural en cuestión y comparación con otras alternativas al alcance del consumidor potencial, para analizar los costes de oportunidad del empleo de recursos públicos en la financiación de un bien particular.

No obstante, Throsby (2003) enfatiza que los cuestionarios diseñados para realizar una estimación de la disposición a pagar por un bien cultural presuponen que los potenciales consumidores están bien informados sobre las características del bien cuyo valor se pretende estimar. Es bien conocido que los bienes culturales son bienes de experiencia, ${ }^{20}$ cuyo gusto se adquiere a través de la experiencia en el consumo $\mathrm{y}$, por tanto, la valoración resultante no necesariamente puede reflejar la verdadera disposición a pagar por parte de los potenciales demandantes.

Seaman (2006, p. 3) además, encuentra que se pueden generar sesgos en los estudios de valoración contingente, que pueden conducir a resultados e interpretaciones erróneos, con referencia a: i) la selección de la muestra (asistentes, población local, población regional) y el diseño del cuestionario (descripción del objeto a evaluar -un festival, un museo-, la construcción del escenario de la valoración, el tipo de pregunta de disposición a pagar adoptada, la elección del vehículo hipotético de pago -impuesto, precio de entrada,

(19) Para mayor información y bibliografía sobre $M V C$ aplicada a los bienes culturales, se puede consultar el número monográfico del Journal of Cultural Economics, v. 27, n. 3-4, nov. 2003.

(20) Nelson (1970, p. 312) identifica dos procedimientos para adquirir información acerca de la calidad, atributos y precios de los bienes, éstos son: i) la búsqueda antes de la compra, y ii) la experiencia después de la compra. En principio, es fácil observar que buena parte de los bienes culturales podrían calificarse como bienes con características de experiencia, dado que, para su disfrute es necesario tener un conocimiento previo de sus atributos y condiciones: una ópera, un concierto de música clásica, danza clásica, arte religioso, cine, etc. 
contribución voluntaria-); ii) el "efecto anclaje" producido por el cuestionario al proporcionar un valor de partida de la disponibilidad a pagar por el entrevistado, su respuesta puede quedar influenciada, "anclada", entre su disposición a pagar a priori y el valor de referencia suministrado; iii) el carácter hipotético de las preguntas puede generar un sesgo hipotético de las respuestas, al sobrestimar la disposición a pagar de los encuestados, ya que no deben desembolsar el dinero en realidad; iv) el incrustar efectos de varias clases puede generar resultados inconsistentes con la teoría económica (por ejemplo, mostrar un exceso de sensibilidad a la existencia de alternativas que resulten irrelevantes).

Otra vez, parecemos llegar a un nudo gordiano, si bien la $M V C$ significa una importante contribución en la aproximación a cuantificar el valor cultural, su desarrollo y aplicación presenta limitaciones. No debemos olvidar que la naturaleza del valor cultural, es decir, la expresión del valor de los bienes culturales en sus significados: estético, espiritual, social, histórico, simbólico, de autenticidad, en el sentido de Throsby (2001, p. 43-44), o, en el sentido de Frey (2000, p. 15-16), en valores de: existencia, prestigio, opción, educación legado, constituye la piedra angular de la relación entre economía y cultura, tanto en el plano económico, como en el socio-cultural. De forma que, las limitaciones que implique su cuantificación no deben hacer perder de vista que la generación de valor cultural contribuye al logro de resultados, no sólo culturales, como también económicos. En este sentido resulta sugerente la afirmación de Throsby (2003, p. 282):

Supongamos que un policy-maker cultural tiene que elegir entre dos proyectos relacionados con la restauración de dos lugares históricos diferentes, cada uno con un coste de capital de 10 millones de dólares. Sólo uno de los proyectos puede llevarse a cabo debido a que la restricción presupuestaria es exactamente esa cantidad y los proyectos no son divisibles. La relación coste/beneficio del proyecto A, incluyendo todos los impactos de mercado y no mercado correctamente medidos, es de 1,1 , para el proyecto $\mathrm{B}$ es de 0,9 . Ahora, [...], una evaluación independiente del valor cultural de los proyectos es obtenida, y demuestra claramente que el valor cultural del proyecto A es bajo y el del proyecto B es alto. Aceptando la validez de estas mediciones, ¿cuál es el proyecto que el hacedor de políticas debe elegir? Un criterio económico sugiere el proyecto A, uno de orden cultural indicaría el proyecto B. [...] El punto de este ejemplo no es sólo el hecho completamente trivial de que las disyuntivas son inevitables en esta vida, sino para avanzar en el más significativo argumento de que el valor cultural, [...], es probable que influya en la toma de decisiones con respecto a los bienes culturales y de esta forma afectar los patrones deseables de asignación de recursos en este ámbito, aunque los beneficios no puedan ser capturados plenamente por medio del análisis económico tradicional. Si esto es así, hay una ardua tarea por delante, para saber si la aplicación de métodos tales como el "Método de Valoración Contingente" y otros enfoques pueden ser extendidos para tener en cuenta las amplias dimensiones que su aplicación al arte y la cultura implican. $\mathrm{O}$, si nuevas técnicas de medición, quizás adaptadas de otras áreas, necesitan ser desarrolladas. 
Willis y Snowball (2009), cómo alternativa a la $M V C$, aplican la metodología CE al South African National Arts Festival. La mencionada técnica está fundamentada en la teoría de Lancaster (1966), la cual asume que los consumidores demandan bienes en función de sus características. En general, los $C E$ parten de una encuesta que descompone las características de un bien e indaga por las preferencias de los consumidores en función de éstas, dado que se incluye el precio y/o coste de cada alternativa en función de los atributos, la disponibilidad a pagar puede ser estimada indirectamente, a través del ordenamiento o elecciones que manifiesten los consumidores, igualmente, permite estimar el valor de no uso (Hanley; Mourato; Wright, 2001).

Los $C E$ apenas se comienzan a aplicar a los bienes culturales y se aprecian como una alternativa que permite calcular la variación compensatoria (la cantidad de dinero que se le puede detraer y/o entregar a un individuo después de un cambio específico en los atributos y características del bien y mantenerlo en su situación original de utilidad) y el cambio en el precio de admisión que se traduciría en una variación porcentual en la asistencia del público a un determinado tipo de producción artística. En principio, estas medidas permiten aproximarse a la valoración económica de algunos componentes del valor cultural.

\section{Argumentos "convincentes" para justificar la financiación pública del arte y la cultura}

De acuerdo con Van Der Ploeg (2006, p. 1200-1204), si existen "argumentos convincentes" que sustentan la financiación con fondos públicos del arte y la cultura:

i)La sensibilización y la educación cultural temprana generan experiencias culturales duraderas en la población infantil, lo que motiva el consumo futuro (cuando adulto) de las artes y la cultura.

ii) Llevar la alta cultura (teatro, ballet, orquestas sinfónicas) en forma conjunta con la cultura popular a sitios públicos como parques, bibliotecas, salones comunales, genera experiencias culturales importantes dadas las condiciones del arte como bien público y no como un bien de lujo.

iii) Los subsidios a la demanda producen un mayor impacto en la generación de actividades artísticas, como puede ser el subsidio a compradores de arte contemporáneo, que posibilita la salida del producto de jóvenes artistas. $\mathrm{O}$, subsidiar las "sillas vacías" en las primeras representaciones de artes escénicas estimula la creación de nuevas obras.

iv) Muchos de los bienes culturales tienen características de bien público. Siguiendo la regla de Atkinson y Stern (1974), se puede incentivar la demanda de bienes culturales con subsidios si el coste marginal de los fondos públicos empleados resulta bajo y, adicionalmente, los bienes culturales generen un impacto positivo sobre la actividad económica (por la atracción de turistas, trabajadores cualificados y la revaloración del suelo urbano). 
v) El valor de opción (legado intergeneracional) constituye un fuerte argumento para la subvención pública de las artes, en especial, para la restauración y mantenimiento de sitios históricos, iglesias y valores paisajísticos.

En la actualidad, hay un relativo consenso sobre el incentivo para el consumo cultural que significa la educación artística a temprana edad financiada con fondos públicos. En efecto, se argumenta a favor de la inversión pública en educación artística y cultural como vehículo de formación de capital cultural a la Stigler y Becker (1977). ${ }^{21}$ El mecanismo opera así (Champarnaud; Ginsburgh; Michel, 2008; Lasuén et al., 2005, p. 337):

i) La evidencia empírica indica que la educación artística, afecta positivamente la participación (aumento en la asistencia a eventos) en las artes.

ii) Dentro de la familia los padres educados cuando jóvenes pueden transmitir a los hijos el gusto y pasión por las artes.

iii) Por tanto, la educación pública puede tener un efecto directo sobre la generación de capital cultural sobre los niños y un efecto indirecto vía la transferencia de conocimientos de los padres a sus hijos.

En resumen, la educación artística puede provocar un efecto intenso en la generación de capital cultural por parte de la población, lo que incentiva la participación directa de ésta en diferentes eventos, artes escénicas, museos, patrimonio, etc. En términos de lo que implica la financiación pública de la educación artística para la economía del bienestar, es importante recordar la sugestiva observación de Blaug (2003, p.268):

Al evitar emplear el nombre de economía del bienestar paretiana, basada en la teoría estática del equilibrio general, seremos más proclives a prestar atención a los argumentos de bienestar intertemporal para, por ejemplo, subvencionar la educación artística en las escuelas o financiar la conservación del patrimonio artístico para las generaciones futuras. La economía del bienestar paretiana se fundamenta en el principio de la soberanía del consumidor: cada individuo es el mejor capacitado, podría decirse que el único, para juzgar su propio bienestar. Sin abandonar por completo este principio, se debe resaltar que el arte sólo puede ser apreciado por quienes hayan sido sus consumidores: en otras palabras, la soberanía del consumidor debe ser la del consumidor con experiencia. Si ello supone un juicio de valor, también lo producen las comparaciones interpersonales de utilidad y también, como es evidente, la noción misma de economía del bienestar resulta absurda sin juicios de valor. [...], estamos promocionando un enfoque heterodoxo singular para la evaluación del gasto en arte.

(21) En el caso de los bienes culturales, se dice que su consumo conlleva a la generación de hábito o adicción (McCain, 2003; 2006). Stigler y Becker (1977) y Becker y Murphy (1988) presentan un modelo que en su versión inicial fue planteado para la música, sugieren que el gusto por la música se genera por un capital específico que incrementa su apreciación en el futuro, lo que no implica la formación de hábitos o cambios en los gustos de los consumidores. Este capital, denominado "capital de consumo cultural" es producto de una función de producción doméstica, asociada al bien particular que incorpora la habilidad del consumidor para producir el bien transformado y consiste en el tiempo, experiencia y formación previamente dedicada al consumo del bien. Para un análisis detallado de las hipótesis para modelar la formación del gusto por los bienes culturales, ver LévyGarboua y Montmarquette (1996; 2003). 
Por último, la educación artística de la población infantil tiene dos atractivos potenciales a largo plazo; i) la reducción del apoyo Estatal directo a las artes y la cultura, una vez que los individuos la incorporen en sus funciones de demanda, vía la adicción en el consumo y, ii) trasladar a los consumidores la elección sobre qué actividades apoyar. Esta política cultural presenta beneficios adicionales, en relación con los fallos del gobierno, el comportamiento de las organizaciones culturales frente al mercado, la captura de rentas y que la oferta cultural dé cuenta de las preferencias de la mayoría de la población y no de una elite cultural reducida.

\section{Ampliando los instrumentos de la política cultural. Los derechos de autor ${ }^{22}$}

Una de las características distintivas de los bienes culturales es que contienen, al menos en potencia, algún derecho sobre la propiedad intelectual atribuible a quien o quienes los producen. Desde el trabajo seminal de Landes y Posner (1989), se identifica que los derechos de autor, entendidos como la protección jurídica de la producción y posterior acceso a las obras creativas, representan un claro beneficio; retribuyen al creador del bien permitiendo excluir a los usuarios no autorizados (free-riders), cargando un precio por su uso. ${ }^{23}$

Towse $(2006 ; 2008)$ llama la atención sobre la escasa importancia que, desde la economía de la cultura, se le ha dado a los derechos de autor. En particular, afirma que tanto las subvenciones como los derechos de autor tienen por objetivo ofrecer incentivos para aumentar la producción cultural más allá de lo que el libre mercado puede lograr. En este contexto, los derechos de autor (copyright) se deben incorporar a la política cultural dado que constituyen una fuente de financiación y estímulo de la producción artística.

No obstante, la solución otorgada por los derechos de autor implica una paradoja: la norma que indica un consumo eficiente (precio $=$ coste marginal) significaría producción ineficiente ${ }^{24} \mathrm{y}$, por tanto, un resultado socialmente no

(22) En la tradición anglosajona, el denominado copyright, hace énfasis en los llamados derechos económicos, la retribución económica que exige el titular de los derechos por la explotación y/o el uso de la obra protegida. En la tradición europea, los derechos de autor (droit d'auteur) hacen énfasis en los derechos morales (el derecho irrenunciable e inalienable al titular de ser reconocido en su condición de autor de una obra y el de exigir el respeto a la integridad de la obra o actuación y la no alteración de la misma). En este documento, se recalca el primer énfasis por razones de espacio, sin desconocer la relevancia de los derechos morales.

(23) En Towse y Watt (2008), se compilan los trabajos más relevantes publicados en los últimos diez años sobre la economía de los derechos de autor, y, en Towse, Handke y Stepan (2008), se encuentra la revisión de literatura más reciente sobre el tema.

(24) En términos económicos, los procesos creativos y de innovación en general, en su función de costes, incluyen un alto coste fijo (esfuerzo, investigación, tiempo de ensayos) de producción y un coste marginal muy bajo o nulo. En la fijación de precios, el creador o la empresa no pueden seguir la condición $\mathrm{p}=\mathrm{cmg}$, dado que implicará pérdidas: el creador no vería recompensando su esfuerzo, lo que Landes y Posner (1989) denominan "costes de expresión", y/o la empresa productora no recuperaría el coste fijo, puesto que. la presencia de rendimientos crecientes a escala indican que siempre el coste medio es mayor que el coste marginal 
deseable (Liebowitz; Watt, 2006, p. 515). Resulta clara la paradoja, cobrar un precio cero es eficiente desde la óptica del consumo, ¿por qué excluir a alguien que se puede beneficiar de un bien cuyo uso no implica un coste adicional? pero desde la óptica de la producción un precio cero indica que el bien no se produciría, ¿qué incentivo tendría el productor si, además, debe soportar los costes de la creación? (Baumol, 2003, p. 23-25). En tal sentido, Liebowitz y Watt (2006, p. 515) afirman: "[...] El derecho de autor considera un balance social; la ganancia social que es derivada del disfrute de bienes creativos debe ser equilibrada contra las ineficiencias que implica la fijación de precios por encima del coste marginal".

Asimismo, en la actualidad, el modelo de derechos de autor se enfrenta a un reto con el surgimiento de los procesos de digitalización y la facilidad que implica el rápido (y abaratado) alcance de los hogares al hardware para la realización de copias de productos artísticos (música, libros, videos, cine, fotografía). En este contexto, paralelo al desarrollo de la teoría de los derechos de autor, surge un conjunto de artículos que proponen que la remuneración del trabajo creativo puede ser capturada directamente del mercado por parte de los creadores, sin necesidad de que exista la protección jurídica a través de los derechos de autor (Liebowitz; Watt, 2006; Miceli; Adelstein, 2006; Varian, 2005). En general, el "mundo sin derechos de autor" sugiere el libre acceso a las obras, eliminando la ineficiencia en el consumo provocada por el cobro de un precio positivo. Sin embargo, a pesar de los múltiples modelos sugeridos, ninguno se advierte lo suficientemente amplio para reemplazar el sistema de derechos de autor.

Towse (2006, p. 568-570), en una comparación entre un sistema de derechos de autor y un sistema de subvenciones y ayudas públicas, encuentra que el apoyo estatal se concentra en la alta cultura a través de organizaciones culturales y, poco apoyo le llega a los artistas individuales, en parte explicado por el riesgo moral que supone el apoyo a los individuos. De forma que, un sistema de ayudas y concesiones públicas puede generar riesgos en cuanto a la administración de los recursos:

El inconveniente de los subsidios públicos es que existe algún peligro de desarrollar un concepto oficial de arte por parte del Estado, si las ayudas son administradas por funcionarios públicos. O de amiguismo si el trabajo se delega en los propios artistas (Towse, 2006, p. 569).

Con un sistema de derechos de autor, es el consumidor quien paga el incentivo al trabajo creativo. Mientras que, con un sistema de subvenciones, son los contribuyentes, en un sentido amplio, quienes realizan este pago. No obstante, la amplia duración de los derechos de autor significa una limitación al acceso público de las obras, con un subsidio la obra sería de dominio público desde su creación. De igual forma, existen diferencias entre los sistemas en los incentivos 
derivados para los creadores, que implican riesgo en la calidad de los bienes culturales producidos:

[...] Los subsidios o ayudas son un premio ex-ante, mientras que los derechos de autor (copyright) y los premios son ex-post. Es posible que estos incentivos tengan diferentes efectos: los artistas deben satisfacer criterios previos para obtener una ayuda, lo que podría influir en los planes futuros de creación de obras, y el éxito de ese trabajo [...] puede influir en las posibilidades de una posterior adjudicación. Con los derechos de autor (copyright), la protección del trabajo es automática, independientemente de su calidad y no hay incentivo para producir un trabajo de alta calidad. (Towse, 2006, p. 570)

Otro riesgo se refiere a que el sistema de derechos de autor estimula el trabajo creativo de los artistas a través del mercado. En este caso, la promoción comercial de las obras queda en manos de una industria cultural dominada por oligopolios, los que buscan su propio interés, y no necesariamente los intereses culturales de los países:

Los intereses de la Disney Corporation no necesariamente coinciden con los del público holandés de televisión. Situación que trae a la discusión de los derechos de autor aspectos de elección pública, que nos llevarán muy lejos y no deben ser ignorados (Towse, 2006, p. 570)

Se pone de manifiesto en los anteriores párrafos, que los derechos de autor, como instrumento de política cultural, no están exentos de polémica y críticas por los efectos económicos que su introducción genera, un tema de especial interés se refiere a su excesiva duración (Liebowitz; Margolis, 2005). Sin embargo, la incorporación de los derechos de autor a la política cultural amplía el panorama de los instrumentos disponibles para la financiación de las actividades culturales.

\section{Conclusiones}

En el artículo, se ha revisado una bibliografía amplia acerca del espectro de argumentos para justificar el apoyo con fondos públicos a las artes y la cultura. Desde las preferencias comunitarias (bien de mérito), la presencia de condiciones particulares en la demanda (bienes públicos) o en la oferta ("enfermedad de los costes") y la aportación a la actividad económica y al empleo (EIEC). Se han señalado las limitaciones que presenta cada argumento y a aquellos que pueden resultar convincentes desde la teoría económica. En especial, se destaca a aquellos que se sustentan en los fallos del mercado y expresan el valor cultural asociado a las externalidades positivas que genera el arte y la cultura:

El valor cultural, [...], no tiene ninguna unidad común de medición, es multidimensional y cambiante, y probablemente incluye algunos componentes expresables sólo en términos no cuantificables. Pero las dificultades de su expresión y evaluación no disminuyen su importancia en la identificación de la atención que merecen los fenómenos culturales que lo incorporan o producen (Throsby, 2001, p. 175). 
Resulta claro que las particularidades de los bienes culturales hacen compleja la política cultural: a) ¿cómo valorar el flujo de servicios (externalidades) que generan los bienes culturales (estéticos, espirituales, simbólicos, arquitectónicos, de pertenencia, de legado)? y, b) la soberanía del consumidor, como lo expone Blaug (2003, p. 268) "el arte sólo puede ser apreciado por quienes hayan sido sus consumidores".

En este contexto, dos tareas se aprecian urgentes para la política cultural: i) el refinamiento de los métodos estadísticos y el desarrollo y/o aplicación de nuevas metodologías para la valoración de bienes culturales y poder, aunque parcialmente, cuantificar las externalidades que el arte y la cultura producen y, ii) generar "consumidores con experiencia", dado que sólo el consumidor con experiencia sería el justo juez de lo que se considera arte y cultura y, podría, con sus preferencias y votos monetarios, decidir el conjunto de bienes culturales disponibles en el mercado y cuáles se conservan para las generaciones futuras.

Por último, se encuentra un relativo consenso en las siguientes acciones específicas de política cultural en materia de financiación pública: el fomento a la educación artística en escuelas públicas, con el objetivo de generar capital de consumo cultural a temprana edad; los subsidios a la demanda (para la compra de obras de artistas jóvenes en artes visuales y cubrir las "sillas vacías" de nuevas obras en artes escénicas); el valor de opción para justificar el legado intergeneracional, en especial para el patrimonio (mantenimiento de sitios históricos, grandes monumentos, bibliotecas y museos). En los sectores asociados a las tecnologías de la información y las comunicaciones, los derechos de autor (copyright) significan una alternativa de financiación, pese a la paradoja que implican, por lo que el tema sigue abierto a discusión.

\section{Bibliografía}

ATKINSON, A; STERN, N. Pigou, taxation, and public goods. Review of Economic Studies, n. 41, p.119-128, 1974.

BANFIELD, E. The democratic muse: visual arts and the public interest. New York: Basic Books, 1984.

BAUMOL, W. Performing arts: the permanent crises. Business Horizons, v. 10, n. 3, p. $47-50,1967 a$.

. Macroeconomics of unbalanced growth: the anatomy of urban crisis. American Economic Review, v. 57, n. 3, p. 415-426, $1967 \mathrm{~b}$.

. Performing arts. In: EATWELL, J.; MILGATE, M.; NEWMAN, P. (Ed.). The New Palgrave: a dictionary of economics, 2. London: Macmillan Press, 1987.

Applied welfare economics. In: TOWSE, R. (Ed.). A handbook of cultural

economics. Cheltenham: Edward Elgar Publishing, 2003. p. 20-31. 
BAUMOL, W.; BOWEN, W. On the performing arts: the anatomy of their problems. American Economic Review, Papers and Proceedings, n. 55, p. 495-502, 1965.

Twentieth Century Found, 1966.

Performing arts. The economic dilemma. Cambridge, Ma:

BECKER, G.; STIGLER G. De gustibus non est disputandum. American Economic Review, v. 67, n. 2, p. 76-90, 1977.

; MURPHY, K. A theory of rational addiction. The Journal of Political Economy, v. 96, n. 4, p. 675-700, 1988.

BENTHAM, J. The works of Jeremy Bentham. Chapter I: Art and science-divisions, published under the Superintendence of his Executor, John Bowring, Edinburgh: William Tait, 1838-1843. The Online Library of Liberty, [1843]. 1962. Disponível em: http://oll.libertyfund.org/title/1921/113884 on 2009-05-02. Acesso em: 17 nov. 2008.

BLAUG, M. Where are we now in cultural economics. Journal of Economic Surveys, v. 15, n. 2, p. 123-143, 2001.

Welfare economics. In: TOWSE, R. (Ed.). A handbook of cultural economics. Cheltenham: Edward Elgar Publishing, 2003. p. 476-482.

BORGONOVI, F. Do public grants to American theatres crowd-out private donations? Public Choice, n. 126, p. 3-4, p. 429-451, 2006.

CHAMPARNAUD, L.; GINSBURGH, V.; MICHEL, P. Can public arts education replace arts subsidization? Journal of Cultural Economics, v. 32, n. 2, p. 109-126, 2008.

COWEN, T.; GRIER, R. Do artists suffer from a cost-disease? Rationality and Society, n. 8, p. 5-24, 1996.

CUCCIA, T. Contingent valuation. In: TOWSE, R. (Ed.). A handbook of cultural economics. Cheltenham: Edward Elgar Publishing, 2003. p. 119-131.

CWI, D. Public support of the arts: three arguments examined. Journal of Behavioral Economics, v. 8, n. 1, p. 39-68, 1979.

De MARCHI, N.; GREENE, J. Adam Smith and private provision of the arts. History of Political Economy, n. 37, p. 431-454, 2005.

DEVESA, M. El impacto económico de los festivales culturales. El caso de la Semana Internacional de Cine de Valladolid. Madrid: Fundación Autor, 2006.

DiMAGGIO, P. (2002). Taking the measure of culture: a meeting at Princeton University citado en: MCCARTHY, K.; ONDAATJE, E.; ZAKARAS, L.; BROOKS, A. Gifts of the muse: reframing the debate about the benefits of the arts. Santa Monica, CA: Rand Corporation, 2004.

FREY, B. Has Baumol's cost disease disappeared in the performing arts? Ricerche Economiche, v. 50, n. 2, p. 173-182, 1996.

Superstar museums: an economic analysis. Journal of Cultural Economics, v. 22, n. 2, p. 113-125, 1998.

FREY, B. State support and creativity in the arts: some new considerations. Journal of Cultural Economics, n. 23, p. 71-85, 1999. 
. Art and economics. Heidelberg: Springer-Verlag, 2000.

Public support. In: TOWSE, R. (Ed.). A handbook of cultural economics. Cheltenham: Edward Elgar Publishing, 2003. p. 389-398.

. What values should count in the arts? The tension between economic effects and cultural value. In: HUTTER, M.; THROSBY, D. (Ed.). Beyond price: value in culture, economics, and the arts. New York: Cambridge University Press, 2008. p. 261269.

; MEIER, S. The economics of museums. In: GINSBURGH, V.; THROSBY, D. (Ed.). Handbook of the economics of art and culture. Amsterdam: North-Holland, 2006.

GINSBURGH, V. Economics of art and culture. In: SMELSER, N.; BALTES, P. (Ed.). International Encyclopedia of the Social and Behavioural Sciences. Amsterdam: Elsevier, 2001. p. 758-764.

GOODWIN, C. Art and culture in the history of economic thought. In: GINSBURGH, V.; THROSBY, D. (Ed.). Handbook of the economics of art and culture. Amsterdam: North-Holland, 2006. p. 25-68.

GRAMPP, W. Should the arts support themselves? Economic Affairs, p. 41-45, Dec./Jan. 1986/1987.

. Pricing the priceless: art, artists, and economics. New York: Basic Books, 1989a.

. Rent-seeking in arts policy. Public Choice, v. 60, n. 2, p. 113-121, 1989 b.

HANLEY, N.; MOURATO, S.; WRIGHT, R. Choice modelling approaches: a superior alternative for environmental valuation? Journal of Economic Surveys, v. 15, n. 3, p. 435462, 2001.

HEILBRUN, J. Baumol's cost disease. In: TOWSE, R. (Ed.). A handbook of cultural economics. Cheltenham: Edward Elgar Publishing, 2003. p. 91-101.

; GRAY, C. The economics of art and culture, 2. Cambridge, Ma.: Cambridge University Press, 2001.

HERRERO, L.; SANZ, J.; BEDATE, A. Valoración económica de bienes públicos en relación al patrimonio cultural de Castilla y León: propuesta metodológica y aplicación empírica. Revista de Investigación Económica y Social de Castilla y León, n. 6, p. 6-122, 2003.

HOWSON, S. Lionel Robbins's "Art and the State". History of Political Economy, n. 37, p. $617-646,2005$.

JOHNSON, A.; SACK, A. Assessing the value of sports facilities: the importance of non economic factors. Economic Development Quarterly, v. 10, n. 4, p. 369-381, 1996.

KEYNES, J. Economic possibilities for our grandchildren. In: ESSAYS in persuasion. New York: W.W. Norton \& Co, [1930], 1963. p. 358-373. 
KLAMER, A.; PETROVA, L.; MIGNOSA. Financing the arts and culture in the European Union. Study Policy Department Structural and Cohesion Policies, European Parliament, Brussels, 2006.

LANCASTER, K. A new approach to consumer theory. The Journal of Political Economy, n. 74, p. 132-157, 1966.

LANDES, W.; POSNER, R. An economic analysis of copyright law. Journal of Legal Studies, v. 18, n. 2, p. 325-363, 1989.

LASUÉN, J.; GARCÍA, M.; ZOFÍO, J. Cultura y economía. Madrid: Sociedad General de Autores y Editores/Fundación Autor, 2005.

LEVY-GARBOUA, L.; MONTMARQUETTE, C. A microeconomic study of theatre demand. Journal of Cultural Economics, n. 20, p. 25-50, 1996.

The demand for the arts. In: TOWSE, R. (Ed.). A handbook of cultural economics. Cheltenham: Edward Elgar Publishing, 2003. p. 201-213.

LIEBOWITZ, S.; WATT, R. How to best ensure remuneration for creators in the market for music? Copyright and its alternatives. Journal of Economic Surveys, v. 20, n. 4, p. 513-545, 2006.

; MARGOLIS, S. Seventeen famous economists weigh in on copyright: the role of theory, empirics, and network effects. Harvard Journal of Law \& Technology, v. 18, n. 2, p. 436-457, 2005.

MADDEN, C. Using 'economic' impact studies in arts and cultural advocacy: a cautionary note. Media International Australia, Incorporating Culture \& Policy, n. 98, p. 161-178, 2001.

MARSHALL, A. Principios de economía. Madrid: Aguilar, [1890] 1954.

MAZZANTI, M. Cultural heritage as multi-dimensional, multi-value and multi-attribute economic good: toward a new framework for economic analysis and valuation. Journal of Socio-Economics, n. 31, p. 529-558, 2002.

MCCAIN, R. Taste formation. In: TOWSE, R. (Ed.). A handbook of cultural economics. Cheltenham: Edward Elgar Publishing, 2003. p. 445-450.

. Defining cultural and artistic goods. In: GINSBURGH, V.; THROSBY, D. (Ed.). Handbook of the economics of art and culture. Amsterdam: North-Holland, 2006. p. $147-167$.

MCCARTHY, K.; ONDAATJE, E.; ZAKARAS, L.; BROOKS, A. Gifts of the muse: reframing the debate about the benefits of the arts. Santa Monica, CA: Rand Corporation, 2004.

MICELI, T.; ADELSTEIN, R. An economic model of fair use. Information Economics and Policy, v. 18, n. 4, p. 359-373, 2006.

MILL, J. S. Principios de economía política. Ciudad de México: Fondo de Cultura Económica, [1848], 1943.

MOEN, M. Congress and the national endowment for the arts: Institutional patterns and arts funding, 1965-1994. The Social Science Journal, v. 34, n. 2, p. 185-200, 1997. 
MOGGRIDGE, D. Keynes, the arts, and the state. History of Political Economy, v. 37, n. 3, p. 535-555, 2005.

MOURATO, S.; MAZZANTI, M. Economic valuation of cultural heritage: evidence and prospects. In: DE LA TORRE, M. (Ed.). Assessing the values of cultural heritage. Los Angeles: The Getty Conservation Institute, 2002. p. 51-76.

MULCAHY, K. Cultural policy: definitions and theoretical approaches. Journal of Arts Management, Law and Society, v. 35, n. 4, p. 319-330, 2006.

MUSGRAVE, R. Teoría de la hacienda pública. Madrid: Aguilar, [1959] 1968.

. Merit goods. In: DURLAUF, S.; BLUME, L. (Ed.). The New Palgrave Dictionary of Economics, 3. Hampshire: Palgrave Macmillan, 2008.

NELSON, P. Information and consumer behavior. The Journal of Political Economy, v. 78, n. 2, p. 311-329, 1970.

NESF. The arts, cultural inclusion and social cohesion. Dublin: National Economic and Social Forum (NESF), 2007. (NESF Report, n. 350.

NETZER, D. Nonprofit organizations. In: TOWSE, R. (Ed.). A handbook of cultural economics. Cheltenham: Edward Elgar Publishing, 2003. p. 331-340.

NORDHAUS, W. Baumol's diseases: a macroeconomic perspective. The B.E. Journal of Macroeconomics, v. 8, n. 1, Art. 9, 2008.

O'HAGAN, J. Tax expenditures. In: TOWSE, R. (Ed.). A handbook of cultural economics. Cheltenham: Edward Elgar Publishing, 2003. p. 451-457.

PALMA, L. La controversia entre cultura y mercado. Algunos elementos para la reflexión a propósito de la Convención de la UNESCO sobre la protección y promoción de la diversidad de las expresiones culturales. Cuadernos de Economía de la Cultura, n. 6, p. 71-84, 2006.

PEACOCK, A. Welfare economics and public subsidies to the Arts. The Manchester School of Economic \& Social Studies, v. 37, n. 4, p. 323-335, 1969. Reimpreso en: Journal of Cultural Economics, 1994, v. 18, n. 2, p. 151-161.

2000.

. Public financing of the arts in England. Fiscal Studies, v. 21, n. 2, p. 171-205,

RAUSELL, P. Políticas y sectores culturales en la Comunidad Valenciana: un ensayo sobre las tramas entre economía, cultura y poder. Valencia: Universidad de Valencia, 1999.

RICARDO, D. Principios de economía política y tributación. Ciudad de México: Fondo de Cultura Económica, [1821] 1959.

ROBBINS, L. Art and the state. Politics and economics: Papers in Political Economy. London: Macmillan, 1963.

. Unsettled questions in the political economy of the arts. The Three Banks Review, n. 91, p. 3-19, 1971. Reimpreso en: Journal of Cultural Economics, v. 18, n. 1, p. 67-77, 1994. 
RUSKIN, J. Unto this last: four essays on the first principles of political economy. Electronic Text Center, University of Virginia Library, [1862] 2001. Disponível em: http://etext.virginia.edu/toc/modeng/public/RusLast.html. Acesso em: 12 ago. 2008.

SAMUELSON, P. Diagrammatic exposition of a theory of public expenditure. The Review of Economics and Statistics, v. 37, n. 4, p. 350-356, 1955.

SANDMO, A. Public goods. The New Palgrave Dictionary of Economics. $2^{\text {nd }}$. ed. Edited by N. STEVEN; DURLAUF and LAWRENCE E. BLUME. Palgrave Macmillan, 2008.

SCITOVSKY, T. What's wrong with the arts is what's wrong with society. American Economic Review, v. 62, n. 2, p. 62-69, 1972.

SEAMAN, B. Arts impact studies: A fashionable excess, 1987. Reimpreso en: TOWSE, R. (Ed). Cultural economics: the arts, the heritage and the media industries. Cheltenham: Edward Elgar: 1997. v. 2.

. The economic impact of the arts. In: TOWSE, R. (Ed.). $A$ handbook of cultural economics. Cheltenham: Edward Elgar Publishing, 2003. p. 224-231.

The relationship among regional economic impact models: contingent valuation versus economic impact in the case of cultural assets. Andrew Young School of Policy Studies, Georgia State University, 2006. (Research Paper Series, n. 07-05).

SMITH, A. Investigación sobre la naturaleza y causa de la riqueza de las naciones. Madrid: Alianza Editorial, [1776] 1994.

SNOWBALL, J. Measuring the value of culture. Methods and examples in cultural economics. Berlin: Springer, 2008.

; ANTROBUS, G. Valuing the arts: pitfalls of economic impact studies of arts festivals. South African Journal of Economics, v. 70, n. 8, p. 1297-1319, 2002.

THROSBY, D. The production and consumption of the arts: a view of cultural economics. Journal of Economic Literature, v. 32, n. 1, p. 1-29, 1994.

Economic circumstances of the performing artist: Baumol and Bowen thirty years on. Journal of Cultural Economics, v. 20, n. 3, p. 225-240, 1996.

. Cultural capital. Journal of Cultural Economics, v. 23, n. 1, p. 3-12, 1999.

. Economics and culture. Cambridge: Cambridge University Press, 2001.

. Determining the value of cultural goods: how much (or how little) does contingent valuation tell us? Journal of Cultural Economics, v. 27, n. 3, p. 275-285, 2003.

. Assessing the impacts of a cultural industry. Journal of Arts Management, Law, and Society, v. 4, n. 3, p. 188-204, 2004.

THROSBY, D. Introduction and overview. In: GINSBURGH, V.; THROSBY, D. (Ed.). Handbook of the economics of art and culture. Amsterdam: North-Holland, 2006. p. 3-22.

Art, economics of.. In: DURLAUF, S.; BLUME, L. (Ed.). The New Palgrave Dictionary of Economics, 3. Hampshire: Palgrave Macmillan, 2008.

TOWSE, R. Cultural economics: the arts, the heritage and the media industries. Cheltenham: Edward Elgar Pub, 1997. 2v. 
TOWSE, R. (Ed.). A handbook of cultural economics. Cheltenham: Edward Elgar Publishing, 2003. F276, 2005.

Alan Peacock and cultural economics. Economic Journal, n. 115, p. F262-

. Copyright and artists: a view from cultural economics. Journal of Economic Surveys, v. 20, n. 4, p. 567-585, 2006.

Pub, 2007.

(Ed.). Recent developments in cultural economics. Cheltenham: Edward Elgar

; HANDKE, C.; STEPAN, P. The economics of copyright law: a stocktake of the literature. Review of Economic Research on Copyright Issues, v. 5, n. 1, p. 1-22, 2008.

; WATT, R. (Ed.). Recent trends in the economics of copyright. The International Library of Critical Writings in Economics, Cheltenham, Edward Elgar Pub, n. 224, 2008.

VAN den HAAG, E. Should the government subsidize the arts? Policy Review, n. 10, p. 63-73, 1979.

VAN DER PLOEG, F. The making of cultural policy: A European perspective. In: GINSBURGH, V.; THROSBY, D. (Ed.). Handbook of the economics of art and culture. Amsterdam: North-Holland, 2006. p. 1183-1221.

VARIAN, H. Copying and copyright. The Journal of Economic Perspectives, v. 19, n. 2, p. 121-138, 2005.

WEST, E. Arts vouchers to replace grants. Economic Affairs, p. 9-16, Feb./Mar. 1986.

WILLIS, K.; SNOWBALL, J. Investigating how the attributes of live theatre productions influence consumption choices using conjoint analysis: the example of the National Arts Festival, South Africa. Journal of Cultural Economics, v. 33, n. 3, p. 167-183, 2009. 